Revue

Revue de l'histoire des religions

del'histoire des religions

\title{
Techniques de garde du secret en Islam
}

Techniques of Secrecy in Islam

\section{Orkhan Mir-Kasimov}

\section{OpenEdition \\ Journals}

Édition électronique

URL : https://journals.openedition.org/rhr/7775

DOI : $10.4000 /$ rhr.7775

ISSN : 2105-2573

Éditeur

Armand Colin

Édition imprimée

Date de publication : 1 juin 2011

Pagination : 265-287

ISBN : 978-2200-92720-2

ISSN : 0035-1423

\section{Référence électronique}

Orkhan Mir-Kasimov, «Techniques de garde du secret en Islam », Revue de I'histoire des religions [En ligne], 2 | 2011, mis en ligne le 01 juin 2015, consulté le 21 septembre 2021. URL : http:// journals.openedition.org/rhr/7775; DOl : https://doi.org/10.4000/rhr.7775 


\section{Techniques de garde du secret en Islam}

Qu'est-ce que le secret, pourquoi et comment le garder? Ces trois questions structurent notre article : la notion de "secret métaphysique» concerne l'objet du secret, les parties sur le secret " initiatique » et " politique " contiennent un aperçu des raisons et des techniques de garde du secret en Islam. Cette analyse est suivie par une synthèse de la théorie et des pratiques du secret dans les textes hurüfì, mouvement messianique iranien fondé dans la deuxième moitié du xive siècle.

\section{Techniques of Secrecy in Islam}

What is secrecy, and why and how it should be kept? These three questions determine the structure of this article: the notion of "metaphysical secrecy" is about the object of secrecy and the sections on "initiatory" and "political" secrecy contain an overview of the reasons and techniques of secret-keeping in Islam. This analysis is followed by a synthesis based on the theory and techniques of secrecy in the texts of the Hurufi messianic movement, founded in Iran in the second half of the $14^{\text {th }}$ century. 


\section{SECRET MÉTAPHYSIQUE ET RECHERCHE SPIRITUELLE}

"C'est Lui qui détient les clefs de l'Inconnaissable. Nul autre que Lui ne les connaît» (Cor. $6: 59)^{1}$. Dans l'islam comme dans toute autre religion, le secret est avant tout associé au mystère impénétrable de Dieu. Le Coran, en tant que Parole divine révélée dans une langue humaine, en l'occurrence l'arabe, au prophète Muhammad, en est le rappel permanent. Pour le croyant, le texte coranique est chargé d'un potentiel immense. Certes, il comporte un sens obvie apparent, mais aussi des sens cachés, et ne se prête pas toujours à une lecture littérale. Incitant à chercher les significations plus profondes, il interpelle le vécu et l'expérience personnelle du lecteur' ${ }^{2}$. Selon un hadith (propos attribué au prophète Muhammad) bien connu, le Coran contient en effet sept niveaux de profondeur : « Le Coran a un sens exotérique et un sens ésotérique. À son tour ce sens ésotérique a un sens ésotérique, ainsi de suite, jusqu'à sept sens ésotériques (sept profondeurs de profondeur) ${ }^{3}$. »

Le texte coranique contient par ailleurs lui-même la mention de deux types de discours, l'un clair et univoque, l'autre symbolique, secret, dont les clés d'interprétation sont détenues par Dieu seul :

C'est Lui qui a fait descendre sur toi le Livre : il s'y trouve des versets sans équivoque [litt. « des versets fermes », muhkamāt], qui sont la Mère du Livre, et d'autres versets qui peuvent prêter à des interprétations diverses [litt. « ambigus, au sens changeant » (mutashābihāt)]. Les gens, donc, qui ont au cour une inclination vers l'égarement, mettent l'accent sur les versets à équivoque, cherchant la dissension en essayant de leur trouver une interprétation, alors que nul n'en connaît l'interprétation à part Dieu. Mais ceux qui sont bien enracinés dans la science disent : « Nous y croyons : tout est de la part de notre Seigneur! $»^{4}$.

1. Nous nous appuyons sur la traduction du Coran de Muhammad Hamidullah, Beyrouth, Dar al-Bouraq, 2003.

2. Voir sur ce sujet l'étude classique de Louis Massignon, Essai sur les origines du lexique technique de la mystique musulmane, Paris, Vrin, 1968.

3. Traduction de Henry Corbin, in En islam iranien : aspects spirituels et philosophiques, Paris, Gallimard, 1972, vol. III, p. 217-218.

4. L'italique est nôtre. Une autre lecture de ce verset est également possible: «nul n'en connaît l'interprétation, à part Dieu et les enracinés dans la 
Dans la théologie musulmane l'aspect caché, à jamais transcendant et inconnaissable de Dieu est désigné par les termes comme essence (dhāt), invisible (ghayb), réalité intérieure (bātin) : «C'est le Secret essentiel (sirr dhātī), le Tréfonds (kunh) que seul Dieu connait. Pour cette raison, ce Mystère est préservé des altérités (aghyār) et reste caché aux intelligences ('uqūl) et aux regards $(a b s ̦ \bar{a} r)^{5}$.»

Les hommes ne peuvent connaître de ce Secret que ce que Dieu leur révèle. Un célèbre hadìth qudsī (propos où Dieu parle à la première personne) fait coïncider la révélation et la Création : elles résultent toutes les deux de la même raison principale, qui est le désir divin de se faire connaître : «J'étais un Trésor caché et Je n'étais pas connu. Or j'ai aimé être connu. Je créai donc les créatures et Je les fis connaître par Moi. Alors elles Me connurent ${ }^{6}$. »

La connaissance humaine est ainsi nécessairement limitée. Le Coran ne cesse pas de répéter que Dieu seul connaît tous les secrets de la Création, ceux « des Cieux et de la Terre ». Même les prophètes ne possèdent pas la totalité de la science divine. C'est ainsi que, dans le Coran, Dieu fait dire à Muhammad : " Dis-leur (aux mécréants): "Je ne vous dis pas que je détiens les trésors de Dieu, ni que je connais l'Inconnaissable, et je ne vous dis pas que je suis un ange. Je ne fais que suivre ce qui m'est révélé" " (Cor. $6: 50)$.

La distinction entre, d'un côté, l'aspect transcendant de Dieu, son Essence et, de l'autre, Dieu révélé à travers ses Noms et ses Attributs dans l'ensemble de la Création est commune dans la théologie musulmane ${ }^{7}$. Depuis les premiers siècles de l'islam, la question du

Science ». Cette lecture indiquerait donc l'existence de la catégorie particulière des « enracinés dans la Science » qui détiennent les secrets de l'interprétation des versets « ambigus » du Coran.

5. 'Alī b. Muhammad al-Jurjānī, Kitāb al-ta'rîfăt, traduction de Maurice Gloton, Téhéran, Presses Universitaires d'Iran, 1994, p. 290, définition $n^{\circ} 1170$, «Al-ghayb al-maknūn ou al-ghayb al-mașūn, Le Mystère protégé ou le NonManifesté immuable ».

6. Ibid., p. 330.

7. Nous ne pouvons pas, dans les limites de cet article, analyser les positions des différents courants théologiques concernant la possibilité de connaître l'Essence et les Attributs de Dieu. Pour avoir une idée de la diversité des approches à ce sujet, et les références bibliographiques, voir par exemple Binyamin Abrahamov, « Fahr al-Dīn al-Rāzī on the Knowability of God's Essence and Attributes », Arabica 49/2 (2002), p. 204-230. 
rapport entre l'Essence et les Attributs, celle de la manifestation du Transcendant et de l'Éternel dans le monde créé, visible et périssable, est devenue un des sujets centraux des débats théologiques. En islam où, après un débat acharné, culminant avec l'Inquisition (mihna) au $\mathrm{Ix}^{\mathrm{e}}$ siècle, le Coran en langue arabe est majoritairement considéré comme la Parole de Dieu révélée, cette question a nécessairement reçu une coloration linguistique : comment est-il possible que le Verbe divin s'exprime à travers la parole humaine, comme cela se produit dans les messages prophétiques et dans les livres sacrés ${ }^{8}$ ? Comment les réalités et les vérités transcendantes peuventelles être véhiculées par le langage humain, dont les concepts relèvent du monde empirique?

C'est dans le domaine de la mystique musulmane que l'interrogation sur la nature paradoxale du discours à la fois divin et humain a été particulièrement approfondie. Comment dire l'indicible, décrire l'invisible, exprimer l'inexprimable? Quelle forme doit prendre le discours du secret? Les mystiques cherchaient la réponse en tentant de reproduire l'expérience prophétique, de rétablir le contact direct avec la source de la Révélation. Il en a résulté le shațh, le langage des expressions paradoxales, outrancières, prononcées en état d'extase, d' « ivresse spirituelle », lorsque la présence divine bouleverse l'ego du mystique et s'exprime par sa bouche. L'un des exemples les plus anciens et les plus connus du shath est l'exclamation : "Gloire à moi, combien grande est ma puissance! » d'Abū Yazīd Basțāmī ( $\mathrm{IX}^{\mathrm{e}}$ siècle). Nombre de ces locutions attribuées aux maîtres des premiers siècles de l'islam ont été réunies par Rūzbihān Baqlī Shīrāzī, célèbre mystique iranien $\mathrm{du} \mathrm{XII}^{\mathrm{e}}$ siècle, dans son ouvrage intitulé Sharh -i shathiyyat $t^{9}$. C'est aussi dans l'œuvre de Rūzbihān que nous trouvons un développement significatif de la théorie de l'équivocité intrinsèque de toute manifestation du divin. Cette équivocité est caractérisée par deux aspects exprimés par deux termes, talbīs et

8. Pour les différentes approches du statut du Coran dans la société musulmane voir Alford T. Welch, « Al-Kur'ān », EI2, vol. V, surtout p. 428-429. Pour la mihna voir Martin Hinds, « Mihna », EI2. Sur la question du rapport entre la parole divine et la parole prophétique voir William Graham, Divine Word and Prophetic Word in Early Islam, La Haye-Paris, Mouton Publishers, 1977.

9. Ouvrage édité avec une introduction française par Henry Corbin, Commentaire sur les paradoxes des Soufis (Sharh-e Shathîyât), Téhéran-Paris, département d'Iranologie de l'Institut franco-iranien - Adrien Maisonneuve, 1966. 
iltibās, dérivés de la même racine arabe avec une connotation de « travestissement ${ }^{10} »$.

Outre le langage paradoxal du shath, la mystique musulmane a élaboré, à des époques plus tardives, d'autres types de discours résultant de la confrontation d'un vécu spirituel avec les moyens du langage ordinaire. Un exemple particulièrement intéressant en est la poésie mystique persane. Son langage spécifique, développé dans les ouvrages des grands maîtres, de Sanā'̄ Ghaznavī (m. 1151) à 'Abd al-Raḥmān Jāmī (m. 1492), a atteint son point culminant dans l'œuvre de Ḥāfiz Shīrāzī (m. 1389) significativement surnommé « la langue de l'invisible » (lisān al-ghayb). Graduellement, le langage de la poésie mystique persane parvient à adapter le vocabulaire du langage quotidien le plus ordinaire et celui de la poésie profane au discours portant sur les réalités métaphysiques. Dès lors, cette poésie peut être lue à plusieurs niveaux. Un lecteur non averti la lira au pied de la lettre et restera au niveau de la poésie profane; mais un lecteur avancé dans la pratique spirituelle y découvrira d'autres profondeurs. L'un des textes les plus importants, à la fois poétique et théorique, sur le fonctionnement de cette transmutation sémantique opérée au sein du langage est probablement le Sawānih («Inspirations ») d'Ahmad Ghazālī (m. 1126) ${ }^{11}$. Voici comment cet auteur formule l'idée d'expression des réalités contemplées par le mystique engagé dans la voie de l'Amour divin par les mots du langage ordinaire :

Ces notes [sont] divisées en quelques chapitres relatifs aux significations [que fait naître] l'amour [mystique]. Cependant, le récit de l'amour ne peut pas être contenu dans les mots et dans les expressions [du langage ordinaire], parce que les significations [qu'il contient] sont [comme les] vierges dont la main des mots est incapable d'atteindre le bout du voile. Bien que notre tâche consiste à marier les vierges des significations aux hommes des mots dans l'intimité des chambres de la parole, les expressions dont se compose ce récit [ne] sont [que] des allusions aux différentes significations [ainsi dissimulées] derrière l'incertitude. Mais cette incertitude [n'existe] en réalité que pour celui qui est privé de l'intuition (litt. «goût», dhawq) spirituelle. Le récit

10. Pour la théorie de l'équivocité chez Rūzbihān voir l'étude de Paul Ballanfat, Quatre traités inédits de Rūzbihān Baqlì Shīrāzì, Institut Français de Recherche en Iran, Téhéran, 1998, p. 181-201.

11. Texte persan édité par Helmut Ritter, sous le titre Ahmad Ghazzālī's Aphorismen über die Liebe, Istanbul, Mațba'a-yi Ma'ārif, 1942. Traduit en anglais par Nasrollah Pourjavady, sous le titre Sawāninh, Inspirations from the World of Pure Spirits, Londres, Kegan Paul, 1986. Sur Ahmad Ghazālī et son œuvre, voir aussi Nasrollah Pourjavady, Sulțān-i țarīqat, Téhéran, Agāh, 1358/1979. 
se divise ainsi en deux récits : l'un est celui des [significations auxquelles] font allusion les expressions, l'autre, ce sont les expressions [qui traduisent] les allusions. Au cœur des mots [il y a] les tranchants des épées, mais ils sont invisibles sauf à l'œil de la vision spirituelle. Si donc, dans ces chapitres, il y a les choses incompréhensibles, elles font partie [de ces significations cachées] ${ }^{12}$.

Parallèlement au développement du langage symbolique de la poésie mystique prend forme la conception philosophique du Monde des Images ou, d'après l'expression de Henry Corbin, du « Monde Imaginal » (ālam al-mithāl), celui de l'Imagination créatrice (ālam al-khayāl). Parmi les penseurs qui ont contribué à l'élaboration de cette conception on peut citer des grands noms comme Abū Nasr al-Fārābī (m. 950), Avicenne (m. 1037), Shihāb al-Dīn Yahyà Suhrawardī (m. 1191), Ibn 'Arabī (m. 1240). Le Monde des Images est un monde intermédiaire où se rencontrent les réalités invisibles du monde supérieur et les images du monde sensible. Il peut être atteint au moyen de l'imagination active, une faculté particulière qui est, par rapport aux images du Monde Imaginal, ce que les yeux sont par rapport aux objets du monde physique. L'idée est très proche de celle qu'exprime Ahmad Ghazālī dans le texte que nous venons de citer. Pour reprendre ses termes, nous pouvons dire que c'est dans le Monde des Images qu'a lieu le «mariage » des significations spirituelles invisibles avec les mots et les expressions qui se réfèrent au monde de l'expérience sensible. Cependant, pour les philosophes et mystiques, la signification spirituelle revêtue par les images du monde physique dans le Monde Imaginal n'est nullement arbitraire ou fantaisiste - il s'agit, au contraire, du sens le plus vrai, archétypique et essentiel de ces images, qui reste caché dans le monde sensible derrière les apparences et les conventions. Le mystique n'a ainsi besoin de recourir à aucun « code » ou " chiffre » artificiel pour assigner aux mots du langage ordinaire des acceptions philosophiques ou spirituelles - ces dernières s'y trouvent attachées naturellement et sans aucune ambiguïté pour quiconque possède l'Imagination créatrice, où, comme le dit Aḥmad Ghazālī, le « goût » spirituel.

En ce qui concerne la poésie mystique persane, ce caractère régulier, non-arbitraire de la transposition sémantique du lexique ordinaire opérée au niveau du Monde Imaginal, a rendu possible,

12. Sawānih , édition de Helmut Ritter, p. 2, la traduction française est nôtre. 
à des époques plus tardives, la composition de véritables « dictionnaires » qui spécifient le sens métaphysique du lexique utilisé par les poètes. Voici quelques exemples tirés de l'un de ces « dictionnaires », un texte anonyme intitulé Mir'āt-i 'ushshāq (« Miroir des amoureux »), datant probablement du Xvi ${ }^{\mathrm{e}}$ siècle $^{13}$ :

Geste amoureux: on appelle ainsi l'attention bienveillante que Dieu accorde à l'itinérant, en assurant ainsi les conditions nécessaires pour attirer le coeur de ce dernier vers la Réalité.

Fleur : c'est le résultat de la connaissance qui éclot dans la plaine du cœur du gnostique.

Joyau : on nomme ainsi le sens véridique, que l'itinérant extrait des coquilles de la parole et de l'expression.

Cil : on désigne ainsi la négligence dont fait preuve le gnostique dans ses actes, en ne le soumettant pas à son regard intérieur.

Cloche : c'est la convocation et la direction communiquées aux facultés de l'âme et aux désirs se produisant chez un être humain afin de l'amener à la perception des plaisirs spirituels.

Débauche : on appelle ainsi la délivrance et l'exemption volontaire de l'itinérant de l'ensemble des liens le rattachant à son être relatif.

Dans les exemples que nous venons de citer, les secrets de la Révélation et de la Parole divine ne font l'objet d'aucune démarche volontaire pour être gardés. Dans la mesure où il est impossible de réaliser la portée véritable des propos paradoxaux des mystiques sans avoir vécu l'expérience qui les a engendrés, de pénétrer les significations profondes du langage poétique sans l'imagination active, le secret métaphysique se charge en quelque sorte de sa propre conservation. Il est impossible d'accéder à ce secret sans s'en rendre digne, et Dieu seul, en dernière instance, décide à qui il sera dévoilé. Cette attitude d' " activité passive », lorsque le mystique s'efforce de se purifier et de rester en éveil dans l'attente d'une révélation, peut être illustrée par le verset coranique suivant : «Et ils disent: "Que ne fait-on descendre sur lui (Muhammad) un miracle de son Seigneur?" Alors, dis : "L'inconnaissable relève seulement de Dieu. Attendez donc; je serai avec vous parmi ceux qui attendent" » (Cor. $10: 20)$.

13. Texte persan publié d'après le manuscrit unique du musée asiatique de Saint-Pétersbourg par Evgeni Edouardovitch Bertels, in Суфизм и суфийская литература (« Soufisme et littérature soufie »), Moscou, Naouka, 1965, p. 126178. Sa traduction intégrale en français a été l'objet de notre diplôme de l'École Pratique des Hautes Études : Orkhan Mir-Kasimov, Réflexions sur le lexique technique de la poésie mystique persane, Paris, EPHE, 1997. Un exemplaire est disponible à la bibliothèque de la Section des sciences religieuses. 


\section{Secret initiatique et Homme-Dieu}

Outre la relation directe et personnelle entre Dieu et le chercheur de vérité, l'islam connaît aussi un autre mode d'économie du secret que nous pouvons appeler le « secret initiatique », c'est-à-dire le secret qui n'est pas, même potentiellement, accessible au commun des hommes, mais dont la garde et la transmission incombent à une personne spécialement habilitée. Ainsi, un Guide, investi de connaissances et de pouvoirs extraordinaires, remplit dans cette optique le rôle d'intermédiaire entre Dieu et les croyants qui sont aptes à recevoir la connaissance supérieure.

Certains versets coraniques peuvent en effet être interprétés - et l'ont été au cours de l'histoire par les différents courants islamiques - comme des allusions à ce type de secret, aux personnes ou aux catégories de personnes qui sont investies par Dieu d'un statut ou d'une connaissance particulière et qui peuvent, de ce fait, servir d'intermédiaires entre Dieu et le reste des hommes. Nous avons cité ci-dessus le verset (Cor. 3 :7, cf. supra note 4) dont une des lectures possibles désigne la catégorie des « enracinés dans la science » qui, seuls parmi les hommes, partagent avec Dieu la connaissance de la vraie interprétation des versets ambigus du Coran. Il y a aussi l'épisode où Dieu crée Adam pour être son « lieutenant » (khalīfa) sur terre (Cor. 2 :30-34). Il apprend ensuite à Adam les noms des choses nouvellement créées, en lui enjoignant de transmettre cette connaissance aux anges. Adam remplit ainsi la fonction d'intermédiaire dans la transmission de la connaissance divine, et les anges se prosternent devant Adam, « à l'exception d'Iblīs (Satan) qui refusa, s'enfla d'orgueil et fut parmi les infidèles ». Jésus a également un statut particulier dans le Coran : «Le Messie Jésus, fils de Marie, est un envoyé de Dieu, Son Verbe qu'Il envoya à Marie, et un Esprit venant de Lui » (Cor. 4 :171).

Qui plus est, le Coran contient également tout un lexique donnant une image anthropomorphe de Dieu Lui-même, image corroborée par les hadith (propos remontant au Prophète et à ses Compagnons). Dieu a des mains, des jambes, des pieds, des yeux, des oreilles; Il entend, voit, rit, se met en colère ${ }^{14}$. Certains de ces propos, large-

14. Pour les anthropomorphismes dans la tradition musulmane, voir l'ouvrage de Daniel Gimaret, Dieu à l'image de l'homme: les anthropomorphismes de la sunna et leur interprétation par les théologiens, Paris, Éditions du Cerf, 1977. 
ment commentés, tant dans les débats théologiques que dans la littérature mystique, parlent ouvertement de Dieu se manifestant sous une forme corporelle humaine : les déclarations telles que «Dieu a créé Adam selon Sa forme », et «j'ai vu mon Seigneur sous la forme d'un jeune homme imberbe, aux cheveux frisés, et vêtu d'un costume vert $»$ en sont des exemples ${ }^{15}$.

L'autorité du texte coranique, fondement scripturaire de l'islam, ne pouvait évidemment être mise en doute. Mais l'interprétation du lexique et des propos anthropomorphiques a divisé les théologiens en deux camps opposés, les « littéralistes » et les « anti-littéralistes » selon l'expression de D. Gimaret ${ }^{16}$. Les premiers soutenaient que tous les propos provenant de sources sûres et faisant partie de la Tradition doivent être pris au pied de la lettre, sans essayer de les comprendre ou de les interpréter. Les seconds rejetaient ce point de vue en disant qu'il est impossible d'assimiler Dieu à la Création en admettant qu'Il peut avoir un corps ou des membres semblables à ceux de l'homme. Le lexique anthropomorphique du Coran doit donc être pris dans un sens métaphorique, par exemple, par « main de Dieu » il faut comprendre « puissance de Dieu ». Globalement, c'est la vision « anti-littéraliste » qui a prédominé, en imposant une certaine censure sur les données du Coran et de la tradition prophétique. Pour autant, la position « littéraliste » n'a pas disparu de la pensée islamique. Elle a connu un développement notable dans les courants existant en dehors de l'Islam « officiel», dans la mystique et la philosophie, et est parvenue, sous différentes formes doctrinales, jusqu'à nos jours.

Déjà aux premiers siècles de l'hégire, certains courants islamiques ont développé à l'extrême la thèse de l'anthropomorphisme en actualisant dans le contexte islamique l'idée antique de divinité de l'homme. Il ne s'agit pas évidemment d'un homme ordinaire, mais de l'Homme avec une majuscule, l'homme qui est véritablement l'image, l'aspect visible, connaissable de Dieu, la personnification des Noms et des Attributs divins. Cet Homme-Dieu est ontologiquement le lieu et la forme de manifestation du secret méta-

15. Pour le texte et les variantes de ces traditions, voir D. Gimaret, op. cit., p. 123-137 et 154-165.

16. Cette présentation est évidemment très générale. Pour le problème terminologique de désignation des deux camps, voir par exemple D. Gimaret, op. cit., p. 10. 
physique, l'intermédiaire entre Dieu inconnaissable et le monde créé. Il s'incarne dans les personnages historiques qui sont, de ce fait, les véritables guides de l'humanité.

Selon toute vraisemblance, l'idée de l'Homme-Dieu faisait partie des doctrines anciennes de l'islam chiite, la plus grande et la plus ancienne « minorité » de l'islam. Elle fonde en effet le statut ontologique de l'imām, sage Guide détenteur d'une connaissance initiatique ${ }^{17}$. En tant qu'incarnations de l'Homme-Dieu archétypique, les imāms historiques non seulement possèdent, mais sont euxmêmes, d'après leur création, la connaissance suprême, la Face visible de Dieu. Ils le déclarent d'ailleurs eux-mêmes dans les propos qui leur sont attribués, propos qui rappellent les shathiyāt des mystiques extatiques que nous avons mentionnés plus haut: « Nous sommes l'CEil de Dieu, nous sommes la Main de Dieu, nous sommes la Face de Dieu, nous sommes Son Côté, Son Cœur, Sa Langue, Son Oreille ${ }^{18}$. » Connaître l'imām revient donc à pénétrer le secret de la connaissance divine. Cette idée est exprimée déjà dans les compilations les plus anciennes des propos attribués aux imāms, comme Kitāb bașā̉ir al-darajāt d'al-Ṣaffār al-Qummī (m. 902-3) : «Celui qui nous connaît, connaît Dieu, et celui qui nous méconnaît, méconnaît Dieu ${ }^{19}$. »

La fonction principale des imāms est de préserver l'influx de la connaissance sacrée après la mort du prophète Muhammad et de rendre disponible la signification profonde du message prophétique, sa dimension secrète. Autrement dit, si Muhammad était chargé de faire «descendre » $(\tan z \bar{l} l)$ la lettre de la révélation, la tâche des imāms consiste à la faire « remonter » $\left(t a^{\prime} w \bar{\imath} l\right)$ à son origine dans le Verbe divin. De ce fait, ils possèdent la science de l'interprétation du Coran et des livres sacrés antérieurs, la science de leurs significations originelles telles qu'elles sont contenues dans la Parole de Dieu.

Il est intéressant d'observer que la transmission de la connaissance secrète, s'effectuant dans la lignée des imāms chiites histo-

17. Muhammad Ali Amir-Moezzi, Le Guide divin dans le shī 'isme originel : aux sources de l'ésotérisme en Islam, Lagrasse, Verdier, 1992, particulièrement p. 73-112; idem, « Aspects de l'imāmologie duodécimaine I : remarques sur la divinité de l'imām », Studia Iranica, 25 (1996), p. 193-215.

18. M. A. Amir-Moezzi, « Remarques sur la divinité de l'imām », art. cité, p. 200.

19. Ibid., p. 201. 
riques qui remonte à 'Alī b. Abī Țālib, cousin et gendre du prophète, et à Fāṭima, fille de Muhammad, est souvent décrite en des termes qui évoquent une substance. Cette connaissance est difficile à porter : « Notre cause est difficile, lourde à assumer; seuls le peuvent un Ange du plus haut rang, un prophète envoyé, ou un croyant fidèle dont Dieu a éprouvé le cœur pour la foi ${ }^{20}$. » Elle constitue un secret confié aux imāms par Dieu, qui ne peut être transmis qu'aux fidèles qui sont créés exprès à cette fin, et qui sont seuls à pouvoir le porter. Les autres humains, destinés à l'Enfer, sont incapables d'assimiler ce secret :

Notre cause est difficile... En vérité, nous (les imâms) tenons de Dieu un secret, un secret dont Dieu n'imposa le fardeau à personne d'autre que nous. Puis il nous ordonna de le transmettre. Nous le transmettons. Mais nous n'aurions trouvé personne qui en fût digne, personne à qui confier le dépôt et qui fût capable de le porter, avant que Dieu n'eût créé à cette fin certains hommes qui furent créés de l'argile de Muhammad et de sa postérité... Nous leur transmettons, de par Dieu, ce que nous avons l'ordre de transmettre. Ils l'accueillent et le supportent; leurs cœurs n'en sont pas troublés. Leurs esprits sont en sympathie avec notre secret; ils tendent spontanément à la compréhension spirituelle de ce que nous sommes; spontanément ils s'enquièrent de notre cause. Mais Dieu a créé aussi des gens qui appartiennent à l'Enfer. Nous avons l'ordre de leur transmettre la même chose. Nous la leur transmettons donc. Mais leurs cœurs se renfrognent devant notre secret; ils s'en effarouchent et nous le renvoient avec un refus; incapables de le supporter, ils crient au mensonge. Dieu a mis une empreinte sur leurs cœurs. Leurs langues articulent une part de vérité; ils en énoncent la formule, mais leurs cœurs la rejettent ${ }^{21}$.

Dieu a donc effacé l'enseignement des imāms des mémoires des non-chiites et a ordonné aux imāms de dissimuler leur connaissance de ces gens, ce que les imāms recommandent aussi à leurs disciples $^{22}$. D'autres traditions précisent la condition de compatibilité des substances, des « argiles » (notamment la co-substantialité

20. Propos attribué à plusieurs imāms, traduit par H. Corbin, En Islam iranien, op. cit., vol. I, p. 14.

21. Propos attribués à Ja'far al-Ṣādiq (m. 699 ou 702-765), vi imām, traduit par H. Corbin, En Islam iranien, op. cit., vol. I, p. 51-52, d'après Kitāb riyāẹ aljannān de Faḍlallāh Mahmūd al-Fārsī.

22. Il s'agit apparemment d'une version de ce même propos, différente de celle utilisée par H. Corbin, résumée par Etan Kohlberg, "Taqiyya in Shī‘̄i Theology and Religion », in Hans G. Kippenberg, Guy G. Stroumsa (éd.), Secrecy and Concealment, Leiden-New York-Cologne, Brill, 1995, p. 368-369, d'après Abū Ja'far al-Kulaynī, al-Kāfì, Téhéran, 1375-77/1956-1958, vol. I, p. 402, nº 5. 
entre le corps de l'imām et le cœur du disciple) nécessaire pour la transmission du secret ${ }^{23}$.

La connaissance que possèdent les imāms ne peut donc pas être révélée à tout un chacun, non seulement pour des raisons tactiques et politiques - nous y reviendrons -, mais aussi pour des raisons qui relèvent de la nature même de cette connaissance. Identique, comme nous l'avons dit, à l'être même de l'imām en tant que lieu de manifestation des Attributs divins, cette connaissance est un secret protégé et réservé à une minorité d'initiés. Plusieurs propos attribués aux imāms en témoignent, comme par exemple celui-ci : « Notre doctrine est un secret contenu dans un secret, un secret bien protégé, un secret dont ne profite qu'un secret, un secret voilé par un secret ${ }^{24}$. »

Ce caractère de la doctrine explique pourquoi la discipline de l'arcane (taqiyya) devient une des composantes centrales de la foi chiite. Nous reviendrons sur les manifestations extérieures de la taqiyya.

\section{SECRET INITIATIQUe : DIMENSION POLITIQUE}

Outre les raisons d'ordre ontologique, liées à la nature même de la connaissance sacrée, le maintien du secret en islam s'imposait aussi - et peut-être surtout - pour des raisons d'ordre politique et social. En effet, nombre de grands maîtres de tendance " extatique » ont subi les persécutions. Leurs propos spontanés et paradoxaux s'inscrivaient mal dans une atmosphère marquée par le renforcement de la théologie dialectique avec son appareil rationnel. En 877, Abū'l Ḥusayn al-Nūrī (m. 907) et d'autres soufis bagdadiens ont été appelés à rendre compte de leurs doctrines, surtout sur la question de la relation amoureuse avec Dieu qui a scandalisé certains théologiens (Ghulam Khalil, m. 888). Après le procès et l'exécution de Manșūr

23. $C f$. par exemple le hadith au sujet de la co-substantialité du corps de l'imām et du cœur du fidèle, M. A. Amir-Moezzi, Le Guide divin, op. cit., note 194 p. 96-97; idem, « Seul l'Homme de Dieu est humain. Théologie et anthropologie mystique à travers l'exégèse imamite ancienne », Arabica, XLV/3 (1998), p. 200 et $s q q$.

24. Bașāi ir al-darajāt, propos attribué à Ja 'far al-Șādiq, traduit par M. A. AmirMoezzi, « Notes à propos de la walāya imamite», in Religion discrète, Paris, Vrin, 2006, p. 193. 
al-Ḥallāj (m. 922), figure légendaire de la mystique musulmane, le soufisme est dominé par le courant « sobre », observant strictement la discrétion et le secret ${ }^{25}$. La tendance extatique ne disparaît pas, mais opte désormais pour les formes d'expression plus discrètes, comme le langage de la poésie mystique persane dont nous avons parlé plus haut, où le contenu métaphysique est voilé par l'utilisation du lexique ordinaire tout à fait profane.

Même s'ils la justifiaient, les maîtres de la tendance " sobre " n'approuvaient pas toujours l'attitude des mystiques « extatiques », considérant leur propos comme un signe d'immaturité spirituelle, la divulgation d'une vérité intime qui devait rester entre Dieu et le fidèle. Selon ce point de vue, le devoir de préserver le secret a donc aussi une dimension éthique : le degré spirituel atteint par le mystique peut être reconnu par ceux qui sont parvenus au même degré, mais doit rester invisible aux gens ordinaires. La divulgation publique de ce secret devant des gens qui sont incapables de le comprendre et de l'apprécier à sa juste valeur ne peut mener qu'à des malentendus. Les malāmatī, "gens du blâme », allaient jusqu'à provoquer volontairement la désapprobation sociale et acquérir une mauvaise réputation pour cacher leur perfection spirituelle ${ }^{26}$.

C'est probablement ainsi que le mot shațh, locution théopathique paradoxale, acquière peu à peu, au moins au sein de certains courants de la mystique musulmane, une connotation négative que nous trouvons dans le Livre des définitions d'al-Jurjānī, savant musulman du XIV ${ }^{\mathrm{e}}$ siècle cité ci-dessus :

Al-shath - Le propos risqué. C'est toute parole dont il émane un souffle de sottise ou d'inconstance et de prétention. Ce propos constitue un manquement chez les êtres de réalisation spirituelle. C'est une prétention à un droit que le gnostique exprime sans autorisation divine, par recherche consciente de la renommée ${ }^{27}$.

Dans le milieu des minorités islamiques, notamment dans les différentes branches du chiisme, la nécessité de la préservation du secret pour des raisons politiques s'imposa dès les premiers siècles del'islam.

25. Pour ces procès, voir par exemple Carl Ernst, Words of Extasy in Sufism, Albany, State University of New York Press, 1985, p. 97-117.

26. Sur les malāmatī, voir Abd al-Raḥmān al-Sulamī, Risālat al-malāmatiyya, traduit par Roger Deladrière, Paris, Arléa, 1991.

27. 'Alī b. Muhammad al-Jurjānī, Kitāb al-ta 'rīfāt, op. cit., p. 233, définition $\mathrm{n}^{\circ} 891$. 
L'idée de l'Homme-Dieu, investi d'une connaissance supérieure, et celle de la continuation de la prophétie dans la lignée des imāms chiites qui, de ce fait, étaient considérés par leurs fidèles comme les seuls guides légitimes de la communauté musulmane, constituaient évidemment une menace très réelle pour le pouvoir califal. Même si les imāms historiques s'efforçaient de se tenir à l'écart de la politique - surtout après la mort tragique du troisième imām, al-Ḥusayn, petitfils du Prophète soupçonné d'être impliqué dans un complot contre le calife régnant et tué à Karbala en 680 -, les révoltes chiites secouaient le jeune empire musulman et ont continué à rester l'un des facteurs les plus importants de l'opposition au pouvoir du calife au cours de l'histoire. Le changement de dynastie califale qui se produisit en 750, lorsque les Abbasides renversèrent les Umayyades, s'appuyait largement sur ce mouvement de contestation. L'État fatimide (909-1171), fondé par les chiites de la branche ismaélienne et gouverné par les imāms vivants, prétendait avoir réalisé le gouvernement juste, appelé à remplacer celui du calife de Bagdad. Ce dernier a été en effet destitué, non par les Fatimides, mais par les Mongols qui prirent Bagdad en 1258. Le coup porté à l'islam majoritaire dont le calife fondait la légitimité ouvrit de nouvelles perspectives aux aspirations politiques des divers groupes chiites et crypto-chiites de cette période, dont émergea finalement, au début du XVI ${ }^{\mathrm{e}}$ siècle, la dynastie des Safavides, instaurant le chiisme duodécimain comme religion officielle de l'Iran.

Dans ce contexte de lutte permanente, les chefs des mouvements «minoritaires » étaient confrontés, dès les premiers siècles de l'islam, à la nécessité de dissimuler leurs enseignements, pour éviter à la fois les malentendus théologiques et les persécutions politiques. Dans le chiisme, la notion de taqiyya, désignant la dissimulation intentionnelle de données doctrinales, de croyances et parfois de l'identité des imāms, est devenue, dès l'époque des imāms historiques, un véritable article de foi chiite. Les propos attribués aux imāms sont catégoriques : « Celui qui propage notre tradition n'est pas des nôtres; il nous a tués délibérément, et non pas involontairement »; "La taqiyya est notre religion et celle de nos pères : celui qui n'observe pas la taqiyya, n'a pas de religion ${ }^{28}$. »

28. Pour les références à ce propos, voir E. Kohlberg, « Taqiyya », art. cité, p. 352 et 356 . Le dernier propos est attribué à l'imām Ja'far al-Ṣādiq. 
Cette dissimulation concerne autant la transmission orale de la connaissance que les enseignements écrits. La dissimulation orale consiste essentiellement en une approche sélective des interlocuteurs : la connaissance destinée aux initiés ne doit pas être divulguée aux profanes. La dissimulation des enseignements écrits posait, quant à elle, des problèmes spécifiques, car ces écrits pouvaient tomber entre toutes les mains. D'où l'utilisation de différentes techniques de cryptage, destinées à interdire au premier venu l'accès au contenu des textes, de façon que seules les personnes ayant la clé du code - ou celles suffisamment motivées pour fournir le travail considérable requis pour le déchiffrer-, puissent le lire. Les compilations des anciennes traditions chiites ainsi que des textes alchimiques attribués au légendaire Jābir b. Hayyān, disciple de Ja'far al-Șādiq, contiennent des exemples de la technique appelée «dispersion de la science » (tabdì $d$ al- 'ilm). Cette technique implique la fragmentation et la dispersion intentionnelle des données doctrinales dans différents endroits du texte ${ }^{29}$. Un vocabulaire spécial était utilisé par les auteurs chiites dans leurs commentaires coraniques, pour masquer les sujets de discorde aux yeux de la majorité sunnite ${ }^{30}$. L'utilisation de signes spécifiques, d'écritures anciennes, comme les alphabets nabatéen et sud-arabique est attestée dans certains textes ismaéliens et nuṣayrîn ${ }^{31}$.

S'ajoutant parfois à l'indisponibilité de textes jalousement gardés par les adeptes, l'application de ces techniques de dissimulation est l'une des raisons pour lesquelles les doctrines de certaines minorités musulmanes sont encore très peu connues.

29. Sur cette technique, voir M. A. Amir-Moezzi, Le Guide divin, op. cit., p. $124,307$.

30. Cf. Meir Bar-Asher, Scripture and Exegesis in Early Imāmī Shiism, Leiden-Boston-Cologne, Brill, 1999, p. 113-120.

31. Voir Meir Bar-Asher, "Outlines of Early Ismā'îlī-Fāțimid Qur'ān Exegesis ", Journal asiatique, 296/2 (2008), p. 257-295; Yaron Friedman, The Nușayrī-'Alawiss, Leiden-Boston, Brill, 2010, p. 281-284; Daniel De Smet, « L'alphabet secret des ismaéliens ou la force magique de l'écriture ", in Charmes et sortilèges. Magie et magiciens, Res Orientales, vol. XIV, 2002, p. 51-60; Rudolf Strothmann, Gnosis-Texte der Ismailiten, Vandenhoeck \& Ruprecht, Göttingen, 1943, introduction, p. 60-61 ; idem, Kitābu'l-kashf of Ja'far b. Manșūri'l-Yaman, Oxford University Press, Londres-New York-Bombay, 1952, introduction, p. 1631. Sur l'utilisation de l'écriture secrète dans les ouvrages d'Ibn al-'Arabī (m. 1240), voir également Gerald T. Elmore, Islamic Sainthood in the Fullness of Time, Brill, Leiden-Boston-Cologne, 1999, p. 574-588. 


\section{DisSiMUler POUR RÉVÉLER, BRISER POUR RECONSTRUIRE LE SENS : IDÉE ET TECHNIQUES DU SECRET DANS LES TEXTES HUUUŪFī}

Le mouvement hurūfī, étymologiquement «lettriste» (de harf pl. hurüf, «lettre» en arabe) est un mouvement à tendance messianique fondé en Iran dans la deuxième moitié du XIV siècle par Faḍlallāh Astarābādī (m. 1394) ${ }^{32}$. Comme beaucoup de mouvements similaires qui émergent en Iran à cette époque, le hurufisme combine des éléments provenant de différents courants islamiques, notamment du chiisme et du soufisme.

Le hurufisme a évolué à une époque particulièrement agitée, marquée par une lutte violente entre maisons rivales se disputant des sphères d'influence en Iran. Convaincu de la vocation messianique de son enseignement, Faḍlallāh a cherché à faire accepter ses doctrines par les puissances politiques. Cette stratégie a été poursuivie après sa mort par ses enfants et ses disciples. Outre des prises de parti dangereuses, les hurūfĩ sont entrés en conflit direct avec le clergé « officiel». Ce dernier a toujours eu le dessus : c'est pourquoi la courte histoire des hurūfĩ en tant que courant indépendant est jalonnée de persécutions et de massacres. Selon toute vraisemblance, les techniques du secret que nous trouvons dans les textes hurūfĩ ont été développées, dans ces conditions d'oppression, pour sauvegarder et transmettre la doctrine. Il nous semble utile de rappeler ici ces techniques en rapport avec les types de secret évoqués en amont ${ }^{33}$.

Tout d'abord, le lien avec le secret «métaphysique » est d'une importance capitale pour instaurer l'autorité spirituelle du fondateur : les textes des auteurs hurūfĩ insistent sur le fait que Faḍlallāh détenait son enseignement directement d'une source surnaturelle. À la suite d'une série de rêves à portée initiatique, il a reçu progres-

32. Pour une présentation générale de ce mouvement, voir par exemple Shahzad Bashir, Fazlallah Astarabadi and the Hurufis, Oxford, Oneworld, 2005; Abdülbaki Gölpinarli, «Faḍl Allāh Ḥurūfī », EI2, vol. II (1977), p. 751-754; Hamid Algar, « Horufism », Encyclopaedia Iranica, New York, vol. XII (2004), p. 483-490; Alessandro Bausani, « Hurūfiyya », EI2, vol. III (1975), p. 620-622; Helmut Ritter, «Studien zur Geschichte der islamischen Frömmigkeit - II, Die Anfänge der Hurūfīsekte », Oriens, vol. 7, n 1 (1954), p. 1-54.

33. Pour une description plus précise des techniques de dissimulation dans les textes hurūfī, voir Orkhan Mir-Kasimov, «Étude de textes hurūfĩ anciens : l'œuvre fondatrice de Faḍlallāh Astarābādī », position de thèse, Revue de l'histoire des religions, 226 (2009), p. 246-260. 
sivement la connaissance de la science du $t a$ 'w $\bar{l} l$, soit de l'exégèse au sens large. D'après Faḍlallāh, la science de l'exégèse est fondée sur la connaissance de la signification métaphysique des lettres de l'alphabet, c'est-à-dire sur la perception des formes des lettres comme autant de lieux de manifestation (mazhar) des Phonèmes primordiaux issus du Verbe divin au moment de la différenciation de celui-ci. Dans leur manifestation la plus matérielle, les lettres sont identifiées avec les 28 signes de l'alphabet arabe et les 32 signes de l'alphabet arabo-persan. Conformément à la cosmogonie hurūfì, les formes (șūra) des lettres sont les formes les plus simples, les «briques» dont est composée la forme corporelle de tout objet, qu'il existe effectivement ou qu'il soit seulement possible; tandis que le Nom ontologique de cet objet, le Nom qui constitue sa signification métaphysique profonde ( $m a$ 'nà), est constitué par les phonèmes. Tout objet peut ainsi être littéralement « lu» par quiconque est capable de distinguer les formes des lettres dans la forme de l'objet. Possédant la connaissance du lien qui réunit ces lettres aux phonèmes du Verbe divin, cette personne est à même d'accomplir «l'exégèse » $(t a$ ' $w \bar{l} l)$ de tout ce qui existe, en ramenant les formes corporelles à leurs significations originelles dans le Verbe, Origine première de toute la Création (conformément au sens étymologique du mot $t a$ ' wìl qui est « retour à l'origine »). À plus forte raison, il en va de même pour l'exégèse des livres sacrés : le lien avec le Verbe est, dans ce cas, encore plus explicite puisque ces livres ne sont rien d'autre que l'expression directe du Verbe dans les langages humains. Connaître la signification de chaque lettre séparée dont ils sont composés donne accès au niveau le plus élevé de l'exégèse, aux vérités cachées derrière la signification conventionnelle des mots et des expressions du langage humain ordinaire ${ }^{34}$.

Autrement dit, dans la perspective hurūfī, détenir le secret des lettres de l'alphabet revient à détenir la science d'une exégèse universelle et définitive. On comprend que si cette ambition avait été officiellement reconnue - et les sources confirment que Faḍlallāh jouissait d'une certaine autorité auprès des gouverneurs locaux et

34. Pour une présentation détaillée de l'histoire des hurūfī et de leur conception du $t a$ 'wìl, voir l'introduction accompagnant l'édition du journal des rêves de Faḍlallāh: Orkhan Mir-Kasimov, «Le "Journal des rêves" de Faḍlallāh Astarābādī : édition et traduction annotée », Studia Iranica, 38/2 (2009), p. 249304. 
des princes de son temps -, elle aurait conféré au prétendant un pouvoir quasi illimité. Entre autres, une telle personne aurait pu réfor-

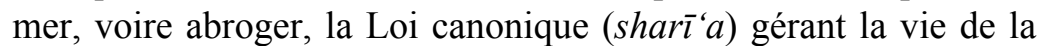
communauté musulmane. L'accès direct au Verbe signifie en effet aussi l'accès direct à la source de la Loi ${ }^{35}$. Ce qui constituait évidemment une atteinte directe à l'autorité des oulémas, dont la réaction, catégorique et violente, ne s'est pas fait attendre dès que les hurūfĩ ont tenté d'introduire leurs doctrines dans les cercles du pouvoir.

C'est probablement pour empêcher l'accès direct à leurs textes à ceux qui y rechercheraient des points d'accusation que les hurūfĩ ont mis en œuvre diverses techniques de dissimulation pouvant servir d'illustration à notre discussion sur le « secret politique ». Nombre de textes hurūfĩ anciens sont rédigés dans un idiome particulier, mélange de persan littéraire et de dialecte local archaïque (celui d'Astarābād, ville natale de Faḍlallāh et de certains de ses disciples). L'utilisation de sigles spéciaux remplaçant quelques expressions courantes ( $c f$. pl. 1) est un autre obstacle capable de décourager le lecteur occasionnel.

Ces techniques constituent le premier échelon de la dissimulation. Le lecteur assidu, ayant assimilé le dialecte et déchiffré les sigles - tache facilitée par ailleurs par les glossaires accompagnant certains manuscrits - est confronté à un autre défi, cette fois d'ordre structural. Certains textes ḥurūfĩ doctrinaux anciens, dont le Jāvdānnāma (le « Livre d'Éternité »), ouvrage principal de Faḍlallāh considéré par ses disciples comme un livre sacré (Jāvdān-nāma-yi ilāhì), présentent une structure fragmentée. Autrement dit, au lieu d'un exposé cohérent et thématiquement ordonné, le lecteur y trouve des paragraphes qui se suivent sans aucun lien logique tout au long de quelque mille pages (500 feuillets environ) du manuscrit. Les passages entre les paragraphes sont marqués par un trait horizontal ou par la «basmala » désignant la formule « Au nom de Dieu, le

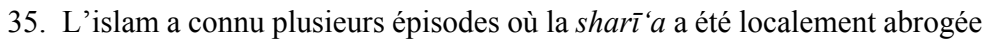
par des mouvements messianiques et antinomistes, qui estimaient la loi religieuse caduque ou inutile en présence d'un guide divinement investi. C'était notamment le cas de certaines communautés ismaéliennes. Voir par exemple Jorunn J. Buckley, « The Nizārī Ismā‘îlīites’ Abolishment of the Sharī‘a During the "Great Resurrection” of 1164 A.D./ 559 A.H. », Studia Islamica, 60 (1984), p. 137-165. Cependant, dans le cas hurūfī, il semble que Faḍlallāh proposait plutôt une réinterprétation de certains préceptes de la loi et du rituel canonique en conformité avec sa doctrine, sans viser une réforme profonde des préceptes eux-mêmes. 
Clément, le Miséricordieux », bismi'llāhi'r-rahmāni'r-rahìm dans le texte du manuscrit de Istanbul Millet Kütüphanesi, Ali Emiri Farsca 920 ( $c f$. pl. 2). Cette technique, rappelant le - et peut-être inspirée $\mathrm{du}$ - tabdìd al-'ilm que nous avons mentionné plus haut, requiert une clé, un principe d'organisation qui permettrait de rétablir la présentation cohérente de ses contenus et d'accéder ainsi à l'exposé doctrinal. Sans nous arrêter sur les détails du procédé de reconstruction que nous avons décrit ailleurs, résumons ses étapes principales ${ }^{36}$.

Il y a d'abord l'idée que la structure fragmentée du Jāvdān-nāma imite celle du Coran. En effet, la discontinuité est l'un des traits caractéristiques du texte coranique. Or, le Jāvdān-nāma contient les passages suggérant de réunir ensemble les versets coraniques concernant le même sujet, mais dispersés dans les diverses endroits du texte, ce qui ne peut ne pas donner au lecteur l'idée d'appliquer le même procédé au texte du Jāvdān-nāma lui-même. Cette idée d'imitation du Coran est par ailleurs confirmée par l'ambition du Jāvdān-nāma, qui se conçoit comme le texte renfermant la science du ta'wīl révélée à Faḍlallāh, celle de l'exégèse définitive du message coranique, de sa reconduction au Verbe divin originel. Les lettres de l'alphabet en général et les lettres mystérieuses du Coran en particulier jouent, nous l'avons dit, un rôle principal dans cette reconduction.

Ensuite, plusieurs éléments confirment que les disciples de Faḍlallāh étaient conscients de l'énigme que représentait la fragmentation du Jāvdān-nāma. La littérature hurūfĩ compte en effet plusieurs ouvrages qui tentent de donner un exposé plus systématique des contenus du Jāvdān-nāma. Une note anonyme placée à la fin du manuscrit de la British Library Oc. Or. 5957 donne une description détaillée de six chapitres dans lesquels il faudrait répartir les passages de l'ouvrage pour l'organiser thématiquement ${ }^{37}$.

Mais même en supposant la connaissance préalable du procédé de réorganisation du texte, la technique de fragmentation imposait un effort considérable à quiconque voulait prendre connaissance

36. Pour le Jāvdān-nāma et les méthodes de la reconstruction de ses contenus fragmentés, voir Orkhan Mir-Kasimov, Étude de textes hurūfí anciens : l'œuvre fondatrice de Fadlallāh Astarābāà̄, thèse de doctorat, École Pratique des Hautes Études, Paris, 2007; idem, "Jāvdān-nāma », Encyclopaedia Iranica, vol. 14/6 (2008), p. 603-605.

37. C'est ce plan, quelque peu modifié afin de mieux l'adapter aux contenus du Jāvdān-nāma, que nous avons suivi dans notre thèse de doctorat. 
des enseignements hurūfī, effort qui garantissait l'ouvrage contre toute interprétation fortuite.

Qui plus est, la technique de fragmentation comporte probablement aussi une dimension « initiatique ». D'une part, comme nous l'avons vu, cette technique limite l'accès aux contenus doctrinaux à une minorité d'individus qui, en l'absence d'enseignement oral du maitre, souhaiteraient entreprendre la reconstruction du puzzle des passages fragmentés. D'autre part, le texte même du Jāvdān-nāma établit un lien entre la fragmentation d'un texte et l'accès à son sens le plus profond, c'est-à-dire entre la fragmentation et le procédé du ta'wìl.

Cette dernière idée est développée dans le commentaire de l'épisode biblique des Tables de la Loi brisées par Moïse (Exode, $32: 19)^{38}$. Se fondant sur sa théorie du $t a$ 'wìl, basée sur la connaissance de la signification ontologique des lettres de l'alphabet, l'auteur propose une interprétation originale de cet épisode. Selon cette interprétation, Moïse a brisé les Tables de la Loi parce qu'il voulait faire surgir les lettres qui les composent. Rappelons que les lettres isolées sont, selon Faḍlallāh, les « lieux de manifestation » des Phonèmes primordiaux du Verbe divin et, en tant que tels, les points de départ de l'exégèse définitive comprise comme la reconduction de la parole de la révélation formulée en une langue humaine à la parole divine originelle :

Moïse jeta les tablettes (de la loi) et les brisa, car la perfection est dans ce qui est brisé, pour que la science des 28 paroles se manifeste... Avant le bris, la science des 28 paroles divines ne se manifeste pas dans la table de la création... Si Moïse n'avait pas brisé les tablettes, la science des 28 et 32 paroles ne se serait pas manifestée. (Jāvdān-nāma, ms. British Library Oc. Or. 5957, f. 414b-415a)

Selon cette interprétation, le texte doit nécessairement être brisé pour qu'on puisse accéder à sa signification véritable. Sans pouvoir l'affirmer avec certitude, nous pouvons supposer que la composition fragmentée du Jāvdān-nāma n'est pas seulement une technique de dissimulation "politique », mais exprime aussi une conception philosophique plus profonde de son auteur.

38. Pour une analyse précise des passages du Jāvdān-nāma relatifs à cet épisode voir Orkhan Mir-Kasimov, «Some Specific Features of the Hurūfĩ Interpretation of the Qur'anic and Biblical Episodes Related to Moses », Journal of Qur'anic Studies, 10/1 (2008), p. 21-49. 
La garde du secret en islam, introduite vraisemblablement pour des raisons d'ordre pratique et politique, comme moyen de sauvegarde et de transmission des idées rejetées par "l'orthodoxie », a graduellement évolué vers des formes plus complexes, s'appuyant sur des doctrines théologiques, philosophiques et mystiques. Les enseignements de nombreux groupes islamiques combinent les différents aspects des techniques de garde du secret que nous avons caractérisé, schématiquement, comme "métaphysique», " initiatique », et "politique». Dans les doctrines de certains de ces groupes, comme nous l'avons vu sur l'exemple des hurūfī, l'idée de garde du secret et les techniques concrètes utilisées à cette fin dans les textes semblent découler des prémisses théoriques les plus fondamentales, dépassant largement le cadre de la dissimulation purement pragmatique ${ }^{39}$.

omir-kasimov@iiis.ac.uk

39. Cet article a été rédigé pendant mon séjour à Berlin en tant que chercheur post-doctorant de la fondation Alexander von Humboldt à l'Institut für Islamwissenschaft de la Freie Universität. Je tiens à exprimer ma reconnaissance à ces deux institutions pour les excellentes conditions d'accueil et les moyens de recherche mis à ma disposition. 
P1. 1. Exemples de sigles utilisés dans les textes hurūfî (ms. Istanbul Millet Kütuphanesi, Ali Emiri Farsca 920)

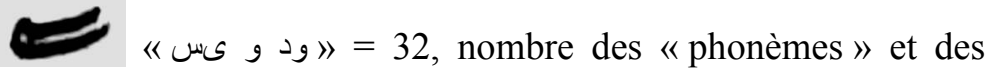
« lettres » primordiales dans la doctrine hurūfî.

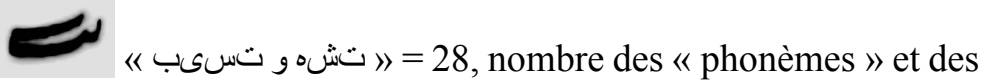
« lettres » primordiales dans la doctrine hurūfî.

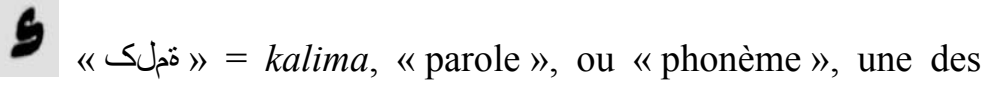
notions fondamentales de la théorie métaphysique du langage développée par Faḍlallāh

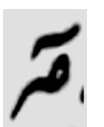

《 $\|$ ڤ Lettre (harf), tandis que $m a$ 'nà, « signification » est associée au Phonème (kalima)

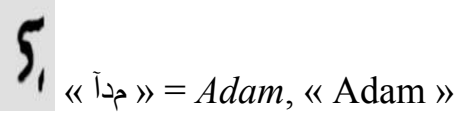

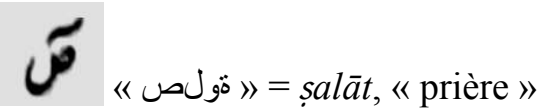

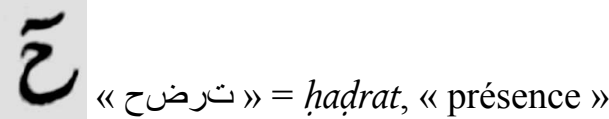

$$
\begin{aligned}
& \text { te. }
\end{aligned}
$$

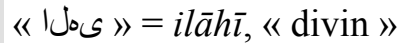


P1. 2. Pages du Jāvdān-nāma, ms. Istanbul Millet Kütüphanesi, Ali Emiri Farsca 920. Les traits horizontaux et les graphies distinctes de la «basmala » indiquent ordinairement les points de rupture entre les fragments.
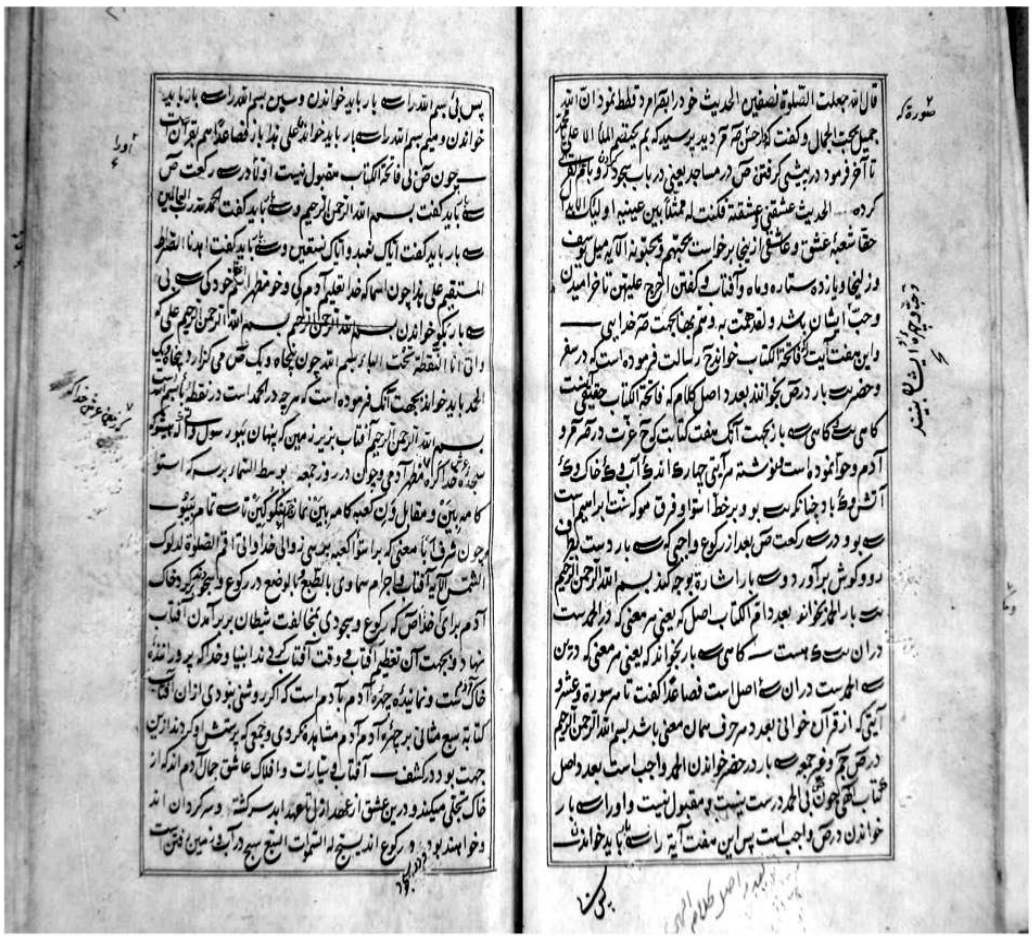
